Ann. Zootech., I979, 28 (2), I65-172.

\title{
Mortalité des Lapereaux au Nid
}

\author{
A. DELAVEAC \\ Avec la collaboration technique de G. LEMOINE et J. P. Coul,Mir \\ Institut Technique de l'Aviculture \\ 28, rue du Rocher, 75008 Paris
}

\section{Résumé}

Le but de ce travail est d'analyser les principales causes apparentes de mortalité des lapereaux durant la période naissance-sevrage, les principales causes de mortalité ont donc été enregistrées, en tenant compte des caractéristiques de chaque lapine, ainsi que de la qualité du nid.

Un comptage a permis de hiérarchiser l'importance des causes de pertes de lapereaux observées entre la naissance et le sevrage. Une analyse en composantes principales montre d'une part, que la mortalité augmente avec l'effectif de la portée, et d'autre part, que la mortalité enregistrée est plus importante si la femelle est saillie jeune pour la première fois. Une analyse factorielle des correspondances montre que les causes de mortalité dues à la mère sont souvent liées à un mauvais nid.

Enfin, les résultats obtenus indiquent clairement que la mortalité des lapereaux a essentiellement lieu durant les deux premières semaines qui suivent la mise bas. Les principales causes de mortalité sont, dans l'ordre : l'abandon par la mère, le cannibalisme et le manque d'allaitement, la taille de la portée et l'âge de la femelle à la première saillie ayant également une incidence notable.

\section{Introduction}

L'élevage cunicole, malgré de récents progrès techniques, est encore soumis à des difficultés nombreuses. Parmi celles-ci, la mortalité des jeunes a une incidence importante sur les résultats économiques de l'élevage.

On observe fréquemment des pertes d'animaux de 25 à $50 \mathrm{p}$. Ioo de l'effectif initial entre la naissance et l'abattage. Mais c'est entre la naissance et le sevrage que l'éleveur enregistre la majorité des pertes, par disparition, soit de quelques lapereaux, soit de la portée entière (LEBAS, I974). Le problème de la mortalité avant sevrage est grave, puisque des pertes moyennes de 4 à 5 lapereaux par portée sont souvent observées; c'est la raison pour laquelle nous avons tenté, dans cette première étude descriptive, d'analyser les principales causes apparentes de mortalité durant la période naissance-sevrage. 


\section{Matériel et méthodes}

\section{Les animaux et les caractéristiques de l'élevage}

Nous avons observé durant trois mois, I 36 portées ayant subi des pertes de lapereaux avant sevrage, soit près de $57 \mathrm{p}$. Ioo des portées contemporaines nées au clapier expérimental de l'ITAVI, à Rambouillet.

Les nids étaient vérifiés chaque matin, à heure fixe; les animaux morts étaient retirés, et la cause apparente de leur mort était alors enregistrée.

Les animaux, de race Néo-Zélandaise pour les femelles et SOLAM type Fauve de Bourgogne pour les mâles, ont été soumis à 16 heures de lumière par jour. Les lapines étaient logées dans une batterie à 3 étages, chaque étage comprenant 2 rangées de 28 cages. La ventilation dynamique du bâtiment était du type surpression, avec un taux de renouvellement de l'air de $2,7 \mathrm{~m}^{3}$ par heure et par kilo de poids vif et un volume d'air disponible par femelle de $I, 5 \mathrm{~m}^{3}$. Les mères ont été nourries à volonté durant la lactation et restreintes à $120 \mathrm{~g}$ par jour jusqu'au vingt-huitième jour de gestation. Les mâles ont été restreints à partir de 3 mois $1 / 2$, 4 mois d'âge, à $\mathrm{I} 20 \mathrm{~g}$ par jour. L'aliment distribué était un aliment classique du commerce.

Les principales caractéristiques de l'élevage, durant la période observée, ont été consignées au tableau I.

\section{Les enregistrements}

Parmi les causes possitles de mortalité, nous avons retenu : l'écrasement, le cannibalisme d̂̀ à la mère, le manque apparent d'allaitement, l'abandon par la mère, la mort de la lapine, la maladie du lapereau. De plus, les animaux jugés chétifs ont été notés comme culots de portée.

Afin d'apprécier la qualité du nid, nous avons attribué des notes comme suit : I, mauvais nid; 2 , nid moyen; 3 , bon nid.

Les données suivantes concernant la lapine ont été prises en considération : l'âge à la première saillie, le nombre de mises bas qui ont précédé l'observation, le pourcentage de mortalité enregistré durant les lactations précédentes, le nombre total d'animaux nés durant les parturitions précédentes, le nombre total de lapereaux nés pour la mise bas observée, le nombre de lapereaux nés vivants pour la mise bas observée, le nombre de lapereaux morts après la mise bas considérée.

\section{Analyses statistiques réalisées}

Cette étude ne se prête pas à un plan expérimental rigide, aussi dans un premier temps avons nous tenté d'analyser les données relatives aux pertes de lapereaux, par des comptages et une illustration graphique des pertes observées. Dans un deuxième temps, nous avons essayé d'analyser les causes de mortalité au nid, en mettant en relation l'ensemble des observations portant sur les caractéristiques des femelles. Pour cette étude, nous avons utilisé les méthodes d'analyses multidimensionnelles. Enfin, dans un troisième temps, afin de relier les causes de morta- 
TABI,EAU I

Principales caractéristiques de l'élevage durant la période d'observation Main characteristics of the rabbit stock during the study

\begin{tabular}{|c|c|c|}
\hline $\begin{array}{l}\text { Variables } \\
\text { Variates }\end{array}$ & $\begin{array}{l}\text { Moyenne } \\
\text { Average }\end{array}$ & $\begin{array}{c}\text { Coefficient } \\
\text { de variation }(\mathrm{p} . \text { Ioo) } \\
\text { Coefficient } \\
\text { of variation }(p . \text { IOo) }\end{array}$ \\
\hline $\begin{array}{l}\text { Age à la première saillie (jours) } \quad \ldots \ldots \ldots \\
\text { Age at first mating (days) }\end{array}$ & I 45 & 26 \\
\hline 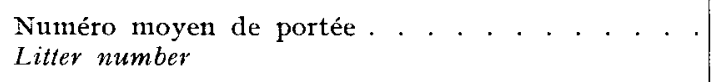 & 2,6 & $5^{2}$ \\
\hline $\begin{array}{l}\text { Mortalité antérieure enregistrée (p. IOO) } \ldots . . \\
\text { Registered previous mortality }(p, I O 0)\end{array}$ & 24 & I I 7 \\
\hline $\begin{array}{l}\text { Nombre d'animaux nés vivants par portée } . \\
\text { Animals born alive per litter }\end{array}$ & 7,2 & 34 \\
\hline $\begin{array}{l}\left.\text { Nonbre de lapereaux morts dans la portée observee ( }{ }^{1}\right) \\
\text { Number of animals dead in the experimental litter }\end{array}$ & 4,1 & 68 \\
\hline
\end{tabular}

(1) I,es portées n'ayant pas eu de pertes sont éliminées de l'échantillon (Litters without death are not considered).

lité apparentes enregistrées à la qualité du nid observée, nous avons effectué un regroupement des principales causes apparentes de mortalité en 2 groupes, le premier concernant les causes que l'on peut imputer à la mère, le second, les autres causes. Une analyse factorielle des correspondances a ensuite été effectuée.

\section{Résultats}

Comme l'indique le tableau 2 , c'est durant les deux premières semaines suivant la mise bas que l'on enregistre l'essentiel de la mortalité; mais c'est surtout pendant les huit premiers jours post-partum que l'on observe une forte mortalité des lapereaux, comme le montre la figure $I$.

Parmi les causes de mortalité, sur 307 lapereaux trouvés morts, on enregistre (tabl. 3) : 


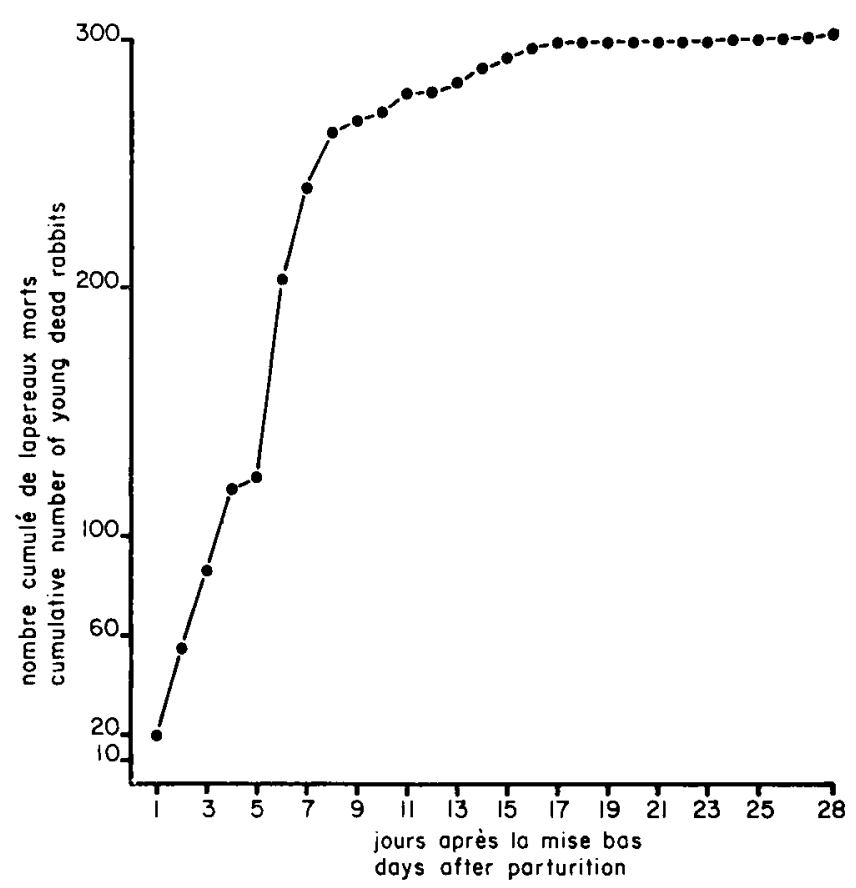

FIG. I. - Répartition quotidienne de la mortalité entre o et 28 jours.

I)aily distribution of mortality from o to 28 days of age.

TABLEAU 2

Répartition hebdomadaire de la mortalité entre o et 30 jours Daily distribution of mortality from o to 30 days of age

\begin{tabular}{|c|c|c|c|c|}
\hline $\begin{array}{l}\text { Semaines d'âge } \\
\text { post-partum } \\
\text { A get-recks } \\
\text { aflcr lirth }\end{array}$ & $\begin{array}{c}\text { I re } \\
(0-7 \text { jours }) \\
I s t \\
(0-7 \text { days })\end{array}$ & $\begin{array}{c}2^{\mathrm{C}} \\
\left(8-14^{\text {jours }}\right) \\
2 ; \mathrm{d} d \\
(8-1+\text { deys })\end{array}$ & $\begin{array}{c}3^{\prime \prime} \\
\left(15-22^{3} \text { jours }\right) \\
3^{\gamma d} \\
(15-22 \text { days })\end{array}$ & $\begin{array}{c}4^{e} \\
\left(23-30^{\circ} \text { jours }\right) \\
4^{t h} \\
(23-30 \text { days })\end{array}$ \\
\hline $\begin{array}{l}\text { Mortalité (p. Iоo) } \\
\text { Mortality rate }(p, \text { Ioo) }\end{array}$ & $7^{8,17} 7$ & 15,63 & $3,5^{8}$ & 0,92 \\
\hline
\end{tabular}

- 96 morts dues à un abandon par la mère; cet abandon concerne le plus souvent des portées entières, ici 8 portées, soit 47 lapereaux, mais aussi parfois quelques animaux seulament d'una portée. L'abandon n'est pas le seul fait des femelles primipares.

- 54 morts par cannibalisme, dont 24 lapereaux seulement provenaient de femelles primipares,

- 36 morts dues à un non allaitement des lapereaux. 


\section{Tableau 3}

Principrles causes de mortalité apparentes observées

Main apparent causes of mortality

\begin{tabular}{|c|c|c|}
\hline $\begin{array}{l}\text { Causes } \\
\text { Causes }\end{array}$ & $\begin{array}{c}\text { Nombre } \\
\text { d'animaux } \\
\text { concernés } \\
\text { Number of animals }\end{array}$ & $\begin{array}{l}\text { p. Ioo d'animaux } \\
\text { par rapport au nombre } \\
\text { de mort total } \\
\text { p. Ioo animals compared } \\
\text { with the total number } \\
\text { of dead animals }\end{array}$ \\
\hline Abandon par la mère (Abandon of young rabbits) & 96 & $3^{\text {I }}$ \\
\hline Cannibalisme (Cannibalism) & 54 & I 8 \\
\hline Manque d'allaitement (Bad nursing). & 36 & 12 \\
\hline 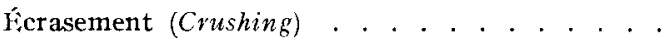 & $2 \mathrm{I}$ & 7 \\
\hline Lapereaux malades (Disease) . . . . . . . . . & I 2 & 4 \\
\hline Culots de portée (Runt animals) . . . . . . . & I I & 4 \\
\hline Mort de la mère (Death of the doe) & Io & 3 \\
\hline Indéterminée ( $U$ nknown). & 67 & $2 \mathrm{I}$ \\
\hline
\end{tabular}

- 2 I morts par écrasement : deux portées entières ont été écrasées, la première comprenait 7 lapereaux, la seconde 8 lapereaux; le reste concerne des animaux isolés au sein du nid. Toutefois, nous n'avons pu déterminer les causes de mortalité pour 67 lapereaux (soit $2 \mathrm{I}$ p. Ioo des animaux morts).

Une analyse en composantes principales portant sur les données caractérisant la lapine, ainsi que sur le nombre de lapereaux nés au cours des mises bas successives (les femelles primipares ont été exclues de cette analyse) a permis de conclure que l'essentiel de notre information de départ peut être résumée par 3 composantes principales, qui expliquent près de $70 \mathrm{p}$. roo de la variabilité totale de la mortalité.

La première composante, qui explique $34 \mathrm{p}$. Ioo de cette variabilité, est caractérisée par l'opposition entre le nombre de lapereaux nés vivants et l'âge à la première saillie; la taille de la portée augmente si la première mise au mâle a été précoce. 


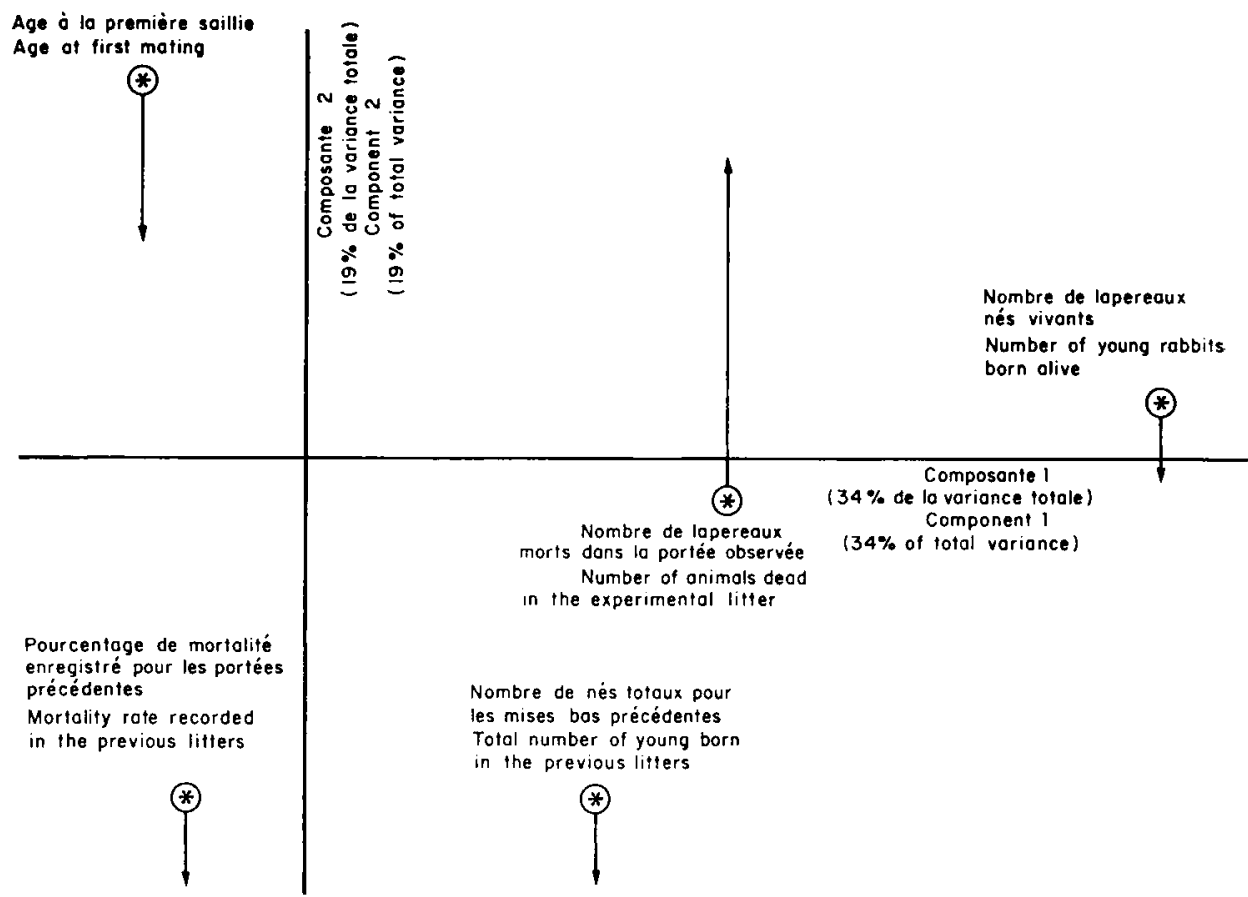

FIG. 2. - Projection des variables sur le plan principal I-2 (analyse en composantes principales) Projection of the variates on the principal plan $1-2$ (principal component analysis)

La deuxième composante (indépendante de la première), qui explique i 9 p. roo de la variabilité totale, montre une liaison entre la mortalité enregistrée au cours des parturitions précédentes et le nombre de lapereaux nés totaux durant ces mises bas : la mortalité augmente avec l'effectif de la portée. De plus, on observe sur ce facteur, que la mortalité est d'autant plus importante que l'âge à la première saillie a été précoce (figure 2).

La troisième composante (indépendante des deux premières), qui explique I6 p. Ioo de la variance totale, indique clairement que la mortalité enregistrée dans la portée observée est d'autant plus importante que la femelle a été saillie jeune pour la première fois (opposition très nette sur cette composante de ces deux variables).

Une analyse factorielle des correspondances ayant pour but de relier les causes de mortalité apparentes enregistrées et la qualité du nid, montre que tous les facteurs de mortalité que l'on peut imputer à la mère sont associés à une mauvaise qualité du nid (figure 3 ). De plus, il ressort de cette analyse que les causes de la mort sont souvent les mêmes pour les lapereaux d'une même portée.

NOTE POUR LA LECTLRE DIS FIGCRES 2 H'T 3

Les valeurs des variables supérieures à zéro portées sur la composante 3 sont indiquées par une flèche ascendante $(\uparrow)$ et les valeurs inférieures à zéro, par une flèche descendante $(\downarrow)$. La longueur de la flèche est proportionnelle à la valeur de la projection de ces variables sur la composante 3 .

The values of the variates $>0$ mentioned on component 3 are shown by an ascending arrow $(\uparrow)$ and the values $<o$, by a desceilding arrow $\left(\begin{array}{l}b \\ \downarrow\end{array}\right)$

The arrowds lenght is proportional to the value of these variate's projection on component 3. 


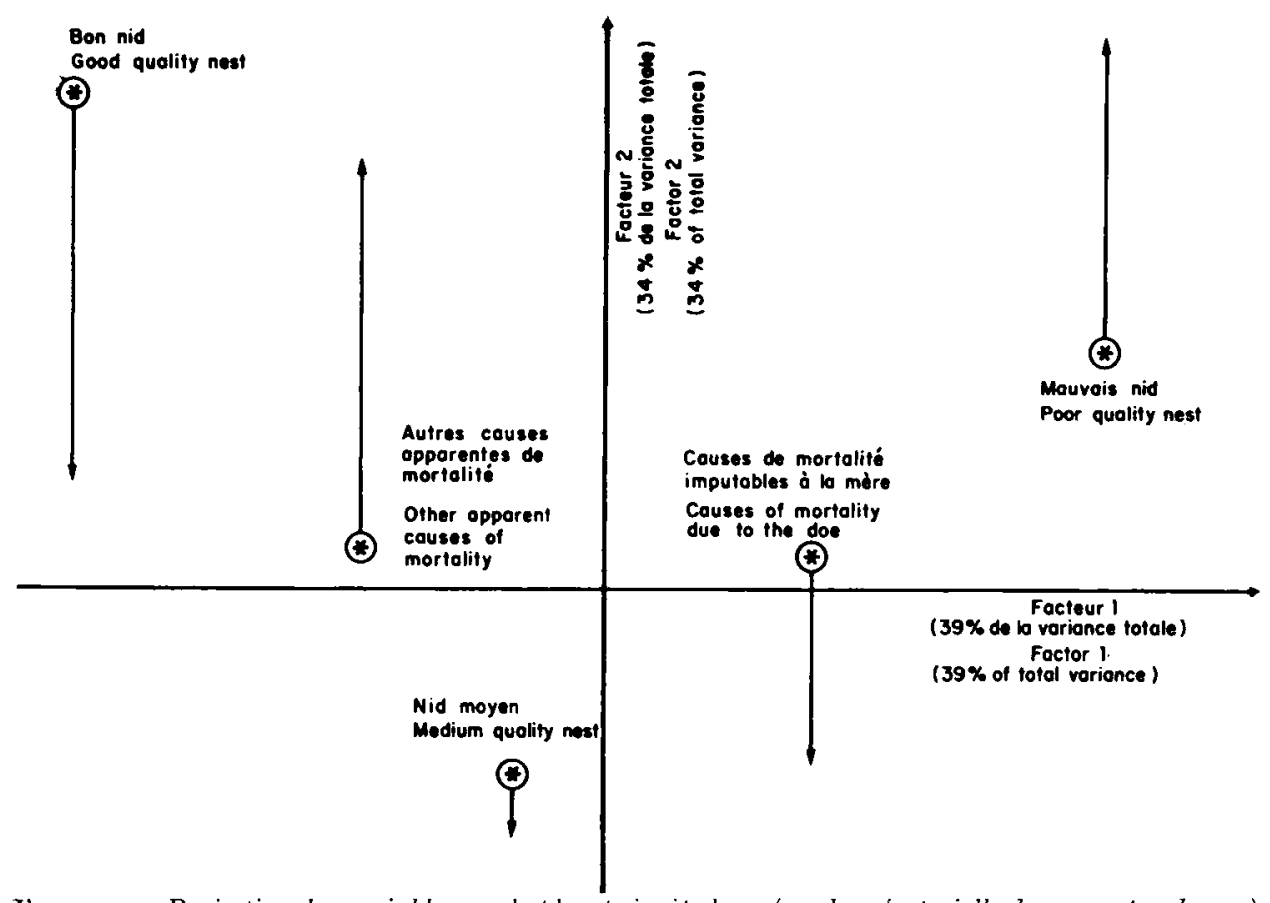

FIG. 3. - Projection des variables sur le plan principal I-2 (analyse factorielle des correspondances) Projection of the variates on the principal plan $1-2$ (Jactorial analysis of the correspondances)

\section{Discussion et conclusion}

Cette étude est avant tout un travail préliminaire, dont la finalité est principalement la mise au point d'une méthodologie d'observation, permettant d'appréhender l'un des problèmes importants de l'élevage cunicole. En effet, nous avons uniquement analysé des causes apparentes de mortalité et de plus, de nombreux lapereaux sont morts sans que l'on puisse déterminer la cause de cette disparition. Toutefois, à notre connaissance, aucune étude n'a été publiée sur ce sujet. I1 conviendrait cependant de pratiquer des autopsies systématiques sur les lapereaux morts et de procéder à des analyses bactériologiques. Ces deux méthodes d'approche demeurent néanmoins difficiles à mettre en application. En effet, 1'autopsie, pour être efficace, doit toujours être effectuée très vite après la mort de l'animal, ce qui n'est matériellement pas possible dans tous les cas (mortalité nocturne par exemple). Quant à l'analyse bactériologique, elle nécessite un acheminement extrêmement rapide vers un laboratoire spécialisé, ce qui pose également de nombreux problèmes pratiques.

En ce qui concerne la répartition de la mortalité des lapereaux en fonction de leur âge, on retrouve les données énoncées par LEBAS (I974) et CHOQUET (I976), à savoir une importante mortalité durant les deux premières semaines post partum, et surtout durant les 7 à 8 premiers jours suivant la naissance. 
La mortalité au nid semble aussi liée à la taille de la portée; des portées importantes sont l'objet d'une mortalité également élevée. VAN DEN BROECK et I AMPO (I975) avaient noté que les pertes les plus grandes affectent les portées de moins de 5 lapereaux et les portées de plus de I3 lapereaux.

Enfin, il convient de prendre en compte l'incidence de l'âge à la première saillie sur la mortalité. Cette donnée précieuse doit toutefois être confirmée par des travaux complémentaires.

En conclusion, il semble que dans notre élevage expérimental, les trois principales causes de mortalité soient : l'abandon par la mère, lecannibalisme etle manque d'allaitement des lapereaux. De plus, on observe une influence de la taille de la portée et de l'âge à la première saillie sur la mortalité des lapereaux.

Accepté pour publication en février 1979 .

\section{Remerciements}

Nous tenons à remercier Mme Luce TAsswcorkr, du Laboratoire de Biométrie de Jouyen-Josas, pour les conseils prodigués a fin de réaliser les analyses statistiques.

\section{Summary}

\section{Mortality of young rabbits in the nest}

The purpose of this study was to analyse the main apparent causes of mortality occurring between birth and weaning in young rabbits. The mait causes of mortality were registered taking into account the characteristics of each doe and the quality of the nest.

The daily distribution of mortality from o to 28 days clearly showed that mortality mainly occurred during the fortnight following birth. The main causes of nortality were: the abandon of the young rabbits, cannibalism and a bad nursing.

A principal component analysis showed that the mortality rate increased with the size of the litter. Moreover this mortality rate was higher if the female was young at first mating.

Another multivariate analysis (factorial analysis of the correspondances) showed that the causes of mortality due to the doe were often connected with a nest of bad quality.

\section{Références bibliographiques}

Choquet B., 1976. Étude préliminaire du comportement maternel de la lapine; comparaison entre types de boîtes à nid. Mémoire de Technicien Supéricur, Rambouillet.

I.EBAS F., I974. La mortalité des lapereaux sous la mère; étude monographique ( ${ }^{\mathrm{r}}{ }^{\circ}$ partie). Cuniculture, $1,8-\mathrm{I}$.

VAX DEN BROECK L., IAMPO P., 1975. Eiffets de quelques facteurs non génétiques sur les performances d'élevage des lapins. Arch. Geflügcl., 39, 84-9o. 